\title{
Addition of levalbuterol to a pediatric emergency department automated medication management system does not increase its use
}

\author{
Gregory P. Conners • Daniel P. Hays • \\ Thomas Richardson • Frank L. Zwemer
}

Received: 30 May 2009 / Accepted: 20 October 2009/Published online: 11 December 2009

(C) Springer-Verlag London Ltd 2009

\begin{abstract}
Background Although adding a drug to an emergency department-based automated medication management system is known to increase how frequently it is ordered, little is known about this effect when the added drug does not offer substantial benefit over a substitute drug that was already available.

Aims We studied the effect of adding nebulized levalbuterol to a pediatric emergency department-based automated medication management system that already included albuterol. Methods All completed orders for nebulized levalbuterol or nebulized albuterol from our academic pediatric emergency department were retrospectively identified using a computerized pharmacy database. We compared ordering of these drugs for the year before levalbuterol was added to the
\end{abstract}

Presented in part at the American College of Emergency Physicians Research Forum, 8-9 October 2007, Seattle, WA.

G. P. Conners $\cdot$ D. P. Hays · T. Richardson · F. L. Zwemer Department of Emergency Medicine,

University of Rochester Medical Center,

Rochester, NY, USA

G. P. Conners

Department of Pediatrics, University of Rochester Medical Center, Rochester, NY, USA

D. P. Hays

Department of Pharmacy, University of Rochester Medical Center, Rochester, NY, USA

G. P. Conners $(\square)$

Children's Mercy Hospitals \& Clinics,

2401 Gillham Road,

Kansas City, MO 64108, USA

e-mail: gpconners@cmh.edu automated medication management system, during which it was available only from the hospital central pharmacy via a pneumatic tube system, with the year following its inclusion in the system.

Results There were 6 orders for nebulized levalbuterol and 1,295 orders for nebulized albuterol during the year that levalbuterol was only available from the hospital central pharmacy, and 7 orders for nebulized levalbuterol and 1,108 orders for nebulized albuterol during the year following levalbuterol's inclusion in the automated medication management system. There was no significant difference $(p=0.78)$.

Conclusions Use of nebulized levalbuterol, in relation to that of nebulized albuterol, for which it is a substitute, did not significantly change when it was included in the pediatric emergency department automated medication management system. This may reflect the lack of substantial benefit that levalbuterol offers over nebulized albuterol in managing children in the emergency department.

Keywords Albuterol · Levalbuterol · Prescribing patterns . Emergency department

\section{Introduction}

Many emergency departments use computerized, automated medication management systems to manage their on-site drug inventories. These systems are meant to improve drug storage security, reduce dosing errors, and improve charge capture, among other features. Little is known about the effects of emergency department-based automated medication management systems on emergency provider prescribing patterns. This includes uncertainty about the effects on drug ordering of adding a drug to a previously established automated 
medication management system. One study has shown that adding drugs that were previously available to an emergency department only from a hospital central pharmacy to such an emergency department system resulted in substantial increases in their use [1]. This suggested that, in the fastpaced emergency department setting, making a drug more rapidly accessible influenced emergency practitioners to order it more frequently. The presence of this effect, however, has not been studied for drugs added to an automated medication management system that did not offer substantial benefit (e.g., lower cost, fewer side effects, higher therapeutic efficacy) over a substitute drug already available via the system.

Nebulized levalbuterol, a substitute for nebulized albuterol, has been shown to provide little, if any, additional benefit over nebulized albuterol in managing children in the emergency department, despite adding additional cost [2-6]. Levalbuterol for nebulization was recently added to our pediatric emergency department's automated medication management system, having previously been available only through the hospital central pharmacy. Since albuterol had already been available for several years in our pediatric emergency department's automated medication management system, the addition of levalbuterol provided a "natural experiment" through which we could study the effect, if any, that adding levalbuterol for nebulization to the pediatric emergency department automated medication management system had on its pediatric emergency department use, in relation to albuterol use. As suggested by previous research [1], we hypothesized that the addition of levalbuterol would be associated with a change in the use of levalbuterol as a substitute for albuterol, compared with the period during which it was available exclusively via the less convenient hospital central pharmacy.

\section{Materials and methods}

\section{Study design and setting}

We conducted a retrospective observational study using a preexisting computerized pharmacy database of a large, urban, academic university hospital to identify all completed orders for either nebulized albuterol or levalbuterol for pediatric emergency department patients (age $\leq 18)$ during the study period.

The pediatric emergency department treats over 26,000 pediatric patients annually in a dedicated pediatric emergency department next to an adult emergency department. The large majority are treated by a combination of resident, fellow, or nurse practitioner and attending physicians; occasionally, minimally acute patients may be seen by a nurse practitioner alone. The hospital has a large formulary of available medications, a fraction of which is available in a frequently used, on-site automated medication management system (Pyxis Medstation ${ }^{\mathrm{TM}}$ ) that is conveniently located in the pediatric emergency department. Selection of medications for inclusion in this system is at the discretion of the hospital Pharmacy Department. Medications are dispensed either from the hospital central pharmacy, typically via a pneumatic tube system, or directly from the onsite automated medication management system. In our pediatric emergency department, nurses may obtain medications readily and simply from the automated medication management system; obtaining medications via the hospital central pharmacy requires several additional steps and significantly more time. The computerized pharmacy database includes prospectively collected data on all completed orders for medications dispensed to patients in the pediatric emergency department, from either source, but does not include information regarding the ordering provider.

Levalbuterol for nebulization was added to our pediatric emergency department's automated medication management system in December 2005, having previously been available only through the hospital central pharmacy. Albuterol for nebulization had been and continued to be available from the pediatric emergency department automated medication management system during the entire study period. The 2-year study period (from December 2004 through December 2006) consisted of the year before and the year after levalbuterol for nebulization was added to the pediatric emergency department's automated medication management system. During the first year, levalbuterol for nebulization was available to pediatric emergency department patients only from the hospital central pharmacy via pneumatic tube. During the second year, levalbuterol was available either through the automated medication management system or the hospital central pharmacy. Levalbuterol metered-dose inhalers were not on the hospital formulary during the study period. Albuterol was available from the automated medication management system in both for nebulization and metered-dose inhaler form during the entire study period. We compared only the use of albuterol for nebulization to use of levalbuterol for nebulization for this study. The inclusion of levalbuterol to the pediatric emergency department's automated medication management system was widely publicized among the pediatric emergency department's physician, nurse practitioner, and nursing staff by the emergency department lead pharmacist, who added the drug to the emergency department system at the request of an individual pediatric emergency department attending physician. Comparative information on drug efficacy, costs, etc. was not specifically distributed. There were no changes in hospital protocol regarding management of acute asthma in children during the study period. As this study had not yet been conceived during the study 
period, practitioners were effectively blinded to all in its aspects.

Outcome measure and data analysis

We compared the number of completed orders for nebulized levalbuterol and for nebulized albuterol in the first year of the study period, during which levalbuterol was available for treatment of pediatric emergency department patients only via the hospital central pharmacy, to the same numbers in the following year, during which levalbuterol was available both from the pediatric emergency department's automated medication management system and from the hospital central pharmacy. Data about medication order completion and patient ages were obtained directly from the computerized hospital pharmacy database; no other patient-specific records were reviewed. We compared the numbers of completed orders with the chi-square test with Yates' correction for continuity.

Our institution's Research Subjects Review Board exempted this study.

\section{Results}

During the first year of the study period, there were 6 completed orders for nebulized levalbuterol and 1,295 for nebulized albuterol. During the second year, there were 7 completed orders for nebulized levalbuterol and 1,108 for nebulized albuterol. We treated a total of 27,754 children in the pediatric emergency department during the first study year and 26,151 children during its second year. Use of nebulized levalbuterol and albuterol were not statistically significantly different between the 2 years (Yates' correction chi-square $=0.078 ; p=0.78$ ). Of interest, the physician who requested the addition of levalbuterol remained on the faculty during the entire study period.

\section{Discussion}

Use of nebulized levalbuterol, in relation to that of nebulized albuterol, for which it is a substitute, did not significantly change when it was included in the pediatric emergency department automated medication management system. Previous research has demonstrated that adding drugs to an emergency department automated medication management system was associated with increased drug ordering [1]. Levalbuterol use may not have increased because levalbuterol offers little, if any, additional benefit over nebulized albuterol in managing children in the pediatric emergency department [2-6]. This would suggest that, while readier access to drugs is an important factor in their selection for use, emergency providers also consider relative benefit when making drug choices.

This study has certain limitations. Although we relied on the accuracy of existing computerized pharmacy records for data on drug use, these records are well maintained at our hospital. Albuterol was available for nebulization from a multi-dose vial during the study period; repeated doses, therefore, could have missed being recorded in the computerized pharmacy record. Levalbuterol, however, was available exclusively in single-dose containers, requiring individual retrieval from our computerized system. Although news of the availability of levalbuterol in the automated medication management system was widely distributed, it could potentially have escaped the notice of some of our providers. A paper-based set of "standing orders" for management of acute asthma in our pediatric emergency department was available during the study period; as these orders included nebulized albuterol but not levalbuterol, this may have biased physicians away from levalbuterol use. However, use of this order page is not required, and it is commonly either not used or altered when the treating provider deems it appropriate. Further, we did not limit our study to patients with asthma, since either albuterol or levalbuterol may be similarly administered to children who are wheezing without a specific asthma diagnosis. Finally, our before-after study design is subject to bias due to a potential environmental or practice change between the two time periods being compared; we are, however, aware of no such relevant changes.

\section{Conclusion}

This study adds an important new facet to the understanding of how the presence of an automated medication management system can affect emergency physician ordering practice. Previous work suggested that readier drug availability via an automated medication management system in itself led to increased use of that drug [1]. This study suggests that lack of advantage over a previously available substitute drug may negate that increase. However, given this study's limitations, we suggest prospective validation of these findings.

\section{References}

1. Conners GP, Hays DP (2007) Emergency department drug orders: does drug storage location make a difference? Ann Emerg Med 50:414-418

2. Milgrom H, Skoner DP, Bensch G, Kim KT, Claus R, Baumgartner RA, Levalbuterol Pediatric Study Group (2001) Low-dose levalbuterol in children with asthma: safety and efficacy in comparison with placebo and racemic albuterol. J Allergy Clin Immunol 108:938-945 
3. Asmus MJ, Hendeles L, Weinberger M, Ahrens RC, Bisgaard H, Lötvall J, O'Byrne PM, Cockcroft DW (2002) Levalbuterol has not been established to have therapeutic advantage over racemic albuterol. J Allergy Clin Immunol 110:325 [letter]

4. Kattan M (2003) Mirror images: is levalbuterol the fairest of them all? J Pediatr 143:702-704
5. Qureshi F, Zaritsky A, Welch C, Meadows T, Burke BL (2005) Clinical efficacy of racemic albuterol versus levalbuterol for the treatment of acute pediatric asthma. Ann Emerg Med 46:29-36

6. Hardasmalani MD, DeBari V, Bithoney WG, Gold N (2005) Levalbuterol versus racemic albuterol in the treatment of acute exacerbation of asthma in children. Pediatr Emerg Care 21:415-419 